\title{
Lo político del lenguaje y los límites de la política lingüística panhispánica
}

\author{
José del Valle* \\ The Graduate Center, City University of New York, EE. UU.
}

\begin{abstract}
Resumen
En este artículo se presenta la glotopolítica y los modelos de articulación del lenguaje y la política que propone. Desde esta perspectiva se analiza la política lingüística panhispánica a partir de un corpus formado por los paratextos de las principales obras codificadoras producidas por la Real Academia Española y la Asociación de Academias de la Lengua Española. El análisis muestra el anclaje ideológico de estas políticas y de los discursos que las exponen en los esquemas conceptuales de la democracia liberal. Finalmente, utilizando las críticas de Chantal Mouffe al pensamiento pospolítico, se contrasta el régimen de normatividad de las academias con lo revelado por dos episodios en los que miembros de la RAE y de la ASALE se ven obligados a polemizar con agentes lingüísticos que operan desde otras instituciones. Y, a raíz del contraste, se concluye exponiendo una limitación fundamental en el discurso de autolegitimación de las academias.
\end{abstract}

Palabras clave: panhispanismo, glotopolítica, normatividad, consenso, pospolítica.

* Para correspondencia, dirigirse a: José del Valle, Ph.D. Program in Hispanic and LusoBrazilian Literatures and Languages (jdelvalle@gc.cuny.edu), teléfono: 1-212-8178410, The Graduate Center, City University of New York, 365 Fifth Avenue, New York, NY 10016, USA. 
The Political IN LANGUAGE AND THE LIMITATIONS OF PANHISPANIC LANGUAGE POLICIES

\begin{abstract}
In this article, we present glottopolitical studies and the models through which this subdiscipline articulates language and politics. From a glottopolitical perspective, we analyze the panhispanic language policy through a corpus of texts that includes the paratextual elements of the principal linguistic works produced by the Royal Spanish Academy and the Association of Academies of the Spanish Language. The analysis shows that these policies and discourse are ideologically anchored in the conceptual structure of liberal democracy. Finally, using Chantal Mouffe's critique of post-political thought, we contrast the academies' linguistic regime with two episodes in which members of RAE and ASALE engaged in polemics with other institutions. This comparison allows us to conclude by exposing the limitations of RAE and ASALE's discourses of selflegitimization.
\end{abstract}

Keywords: Panhispanism, glottopolitical studies, normativity, consensus, post-political.

Recibido: 28/07/14 Aceptado: 24/10/14

\title{
REPRESENTACIÓN DEL LENGUAJE Y GLOTOPOLÍTICA
}

Tal como se adelanta en el anuncio de este número especial, el estudio de las representaciones sociales del lenguaje se puede abordar desde distintas ubicaciones teórico-metodológicas. La glotopolítica, a pesar de la heterogeneidad interna que presenta y que enseguida describiremos, demarca coherentemente una de estas posiciones en tanto que al identificar su objeto le otorga estatus especial precisamente a las representaciones del lenguaje. Hay algo, sin embargo, que singulariza a esta aproximación y es la interpretación de tales representaciones en un contexto definido en términos eminentemente políticos: "Glottopolitique est nécessaire pour englober tous les faits de langage où l'action de la société revêt la forme du politique" [Glotopolítica es necesario para abarcar todos los hechos del lenguaje en los que la acción de la sociedad reviste la forma de lo político] (Guespin y Marcellesi 1986: 5, traducción nuestra). Así justificaban Guespin y Marcellesi, en un 
artículo fundacional, la adopción de un término que confiriera personalidad disciplinaria a un conjunto de proyectos de investigación que, aunque tenían en común el haber articulado el lenguaje con la política, no habían sido objeto conjuntamente de un esfuerzo de teorización.

En un conciso recorrido por la historia del campo, Elvira Narvaja de Arnoux (2000) destaca la formación, a partir de los años cincuenta, de tres corrientes que van conformando los protocolos de una de las subdisciplinas que alimentan el desarrollo de la glotopolítica: la política y planificación lingüística. La primera corriente, cuya aparición se sitúa tras la Segunda Guerra Mundial, responde a condiciones creadas por la división del mundo en áreas de influencia estadounidense y soviética, respectivamente, y por los procesos de construcción nacional asociados a la descolonización de África y Asia. Desde esta perspectiva, se procede a crear un aparato teórico y metodológico que permita describir el perfil sociolingüístico de una comunidad para, a la vez, orientar la toma de decisiones políticas que incidan o afecten tal perfil. Se discute la naturaleza de la estandarización, se debate en torno a categorías tales como esquizoglosia o diglosia y emerge el concepto de bilingüismo armónico como principio ideal de ordenamiento lingüístico que pretende reconocer y compatibilizar el valor de las lenguas de identificación étnica o nacional y la necesidad de promover el conocimiento de las lenguas internacionales ${ }^{1}$.

Una segunda corriente, que cuaja a partir de los años sesenta y que estaría estimulada por los procesos de defensa de los derechos civiles de las minorías, adopta una posición reivindicativa y crítica de la corriente anterior al denunciar el modo en que aquella propone modelos de organización lingüística que perpetúan las jerarquías propias del colonialismo. Desde esta posición, se cuestionará el concepto de bilingüismo armónico y se avanzará la idea de que el contacto es conflicto. Subrayemos este punto -que tendrá relevancia especial al final del presente artículo- citando el modo en que Guespin y Marcellesi lo expresaban:

Il faut remarquer qu'il [le concept de planification linguistique] en va de même du concept de conflit linguistique; toute planification linguistique, dans une société de clases est nécessairement la politique linguistique d'une classe sociale dominante, tout en résultant d'une compromis [Hay que señalar que [el concepto de planificación lingüística] va de la mano del concepto de conflicto lingüístico; toda planificación lingüística, en

\footnotetext{
1 Buenos ejemplos de esta corriente nos los ofrecen, por ejemplo, Haugen (1972) o Kaplan y Baldauf (1997).
} 
una sociedad de clases, es necesariamente la política lingüística de una clase social dominante, $\mathrm{y}$ todo es producto de un compromiso] (Guespin y Marcellesi 1986: 14).

Además de afirmar el carácter conflictual del contacto, desde esta perspectiva se propondrán, en los contextos pertinentes, procesos de estandarización (normativización y normalización) de lenguas históricamente minorizadas que reclaman la introducción de mecanismos de discriminación positiva en su favor ${ }^{2}$.

La tercera corriente que señala Arnoux, que adquiere mayor dimensión ya hacia finales del siglo veinte, está ligada a los procesos políticos y económicos de integración regional, a la formación de redes de interacción transnacionales y a los flujos de capital, gente e información típicamente asociados con la globalización. Se negocian, en esta fase, tensiones entre el valor cultural, político y económico de las lenguas en los mercados lingüísticos regionales, nacionales y globales; las minorías lingüísticas universalizan su reivindicación y engarzan sus discursos con los de los derechos humanos; y se organizan industrias lingüísticas en torno a, por ejemplo, la enseñanza de lenguas extranjeras, la terminología y la traducción ${ }^{3}$.

En todas estas corrientes, el lenguaje se piensa como un ámbito de la vida social susceptible de ser objeto de la acción política, y su estudio se plantea como un encuentro entre una suerte de cartografía sociolingüística y un tipo especial de ingeniería social. Se estudia el perfil sociolingüístico de las comunidades para identificar qué tipo de intervenciones sobre el lenguaje podrán contribuir, en cada caso, a construir una conciencia grupal o nacional, a fomentar transacciones y lealtades internacionales, a facilitar el funcionamiento del aparato del Estado o a crear condiciones para el desarrollo económico. Al revisar el desarrollo de la política y planificación lingüística, nos encontramos ante proyectos de investigación fuertemente ligados a los protocolos de las ciencias sociales de perfil más positivista y a teorías estructurales-funcionales del lenguaje. Se piensan las lenguas como entidades discretas, claramente diferenciadas; se las teoriza como estructuras de varios niveles que corresponden a distintos contextos y cometidos sociales; y esta organización estructural-funcional se proyecta

\footnotetext{
2 Véanse como ejemplo las fuentes citadas en Arnoux (2000); sobre todo en las notas 25 y 26.

Aquí se podría pensar en las actividades y trabajos del Instituto Cervantes sobre el español en el mundo recogidos en los anuarios (http://cvc.cervantes.es/lengua/anuario/). Otra serie de ejemplos son los artículos incluidos en Maurais y Morris (2003).
} 
fractalmente a los espacios plurilingües donde la coexistencia entre lenguas es posible solo gracias a la clara diferenciación de los contextos apropiados para el uso de cada una de ellas ${ }^{4}$.

Ya hemos señalado que el trabajo que se asocia con estas corrientes se identifica por medio de la etiqueta "política y planificación lingüística" y en él la política se concibe como el conjunto de espacios institucionales asociados al gobierno de un territorio - la mayoría vinculados al aparato del Estado- donde se negocia y se ejerce el poder y desde donde se establecen las normas que rigen la vida de la comunidad. Desde esta perspectiva la dimensión política del lenguaje se manifiesta precisamente en esos lugares $\mathrm{y}$ en esas oportunidades en las que se produce una acción consciente y explícita sobre lo que hemos llamado el perfil lingüístico de la comunidad.

Es posible, sin embargo, proponer una articulación más abarcadora de la relación entre lenguaje y política, es decir, proponer una ampliación del espectro de situaciones en que se manifiesta la condición política del lenguaje y su relación con la distribución de poder. Ya Arnoux (2000: 98-99) señalaba en el artículo citado la existencia de una línea de investigación histórica en relación con las políticas lingüísticas que reclamaba la relevancia del lenguaje en múltiples dimensiones de la vida política de las naciones, una relevancia que se manifestaba no solo en la acción política sobre la lengua sino en la pertinencia de la praxis lingüística y metalingüística en el terreno de la política. Esta línea y su voluntad historificadora daba lugar al ingreso al archivo en busca de textos que se iban a tratar no solo como documentos sino también como discursos susceptibles de ser leídos a contrapelo por el ojo glotopolítico y desde una epistemología de la sospecha.

Otra articulación también amplia de lenguaje y política se le debe a John Joseph, quien, en su libro Language and Politics (2006), atiende no solo a la participación del lenguaje en la organización de los asuntos del Estado sino también en la negociación de "cualquier situación en la que haya una distribución desigual del poder" (Joseph 2006: 20). La suya es una propuesta detallada y el título de las secciones en que divide su capítulo introductorio es revelador del espectro de zonas en que se manifiesta la condición política del lenguaje: "The politics of different ways of speaking" [La dimensión política de las diferentes formas de hablar], "The politics of talking to others" [La dimensión política del hablarle a otros], "The politics of what 'the language' is" [La dimensión política de qué es 'la lengua'],

\footnotetext{
4 Marilyn Martin-Jones se refiere a esta línea de trabajo como "perspectiva estructuralfuncional" (1989).
} 
"The politics of which language to speak" [La dimensión política de decidir qué lengua hablar], "The politics of policing the language" [Las políticas de vigilancia del lenguaje], "Language, thought and politicians" [El lenguaje, el pensamiento y los políticos], "Language and choice" [El lenguaje y la capacidad de decisión] y "Conclusion: Language is political from top to bottom" [Conclusión: el lenguaje es político de arriba abajo].

Evidentemente, esta amplia tipología de manifestaciones de la dimensión política del lenguaje configura un objeto de estudio que, en buena medida, coincide con el proyecto expuesto por los ya mencionados Guespin y Marcellesi:

Le concept de glottopolitique ... englobe à la fois les politiques concertées et les relais, conscientes ou inconscients, actifs ou passifs, de ces politiques. Ainsi, le fait glottopolitique va des actes minuscules et familiaux ... jusqu'aux interventions les plus considérables [El concepto de glotopolítica ... abarca a la vez las políticas planificadas y los efectos -conscientes o inconscientes, activos o pasivos-de esas políticas. Así, el hecho glotopolítico incluye desde actos minúsculos y familiares... hasta otros de mayor consideración] (1986: 15).

Para estos autores, glotopolítica ofrece la ventaja de neutralizar la distinción entre langue y parole (1986: 5) e instala el proyecto en una teoría del lenguaje que lo liga siempre, y por medio de una relación dinámica, al contexto. El lenguaje no existe antes del acto en que se manifiesta y se piensa por ello como una práctica social caracterizada por una glotogénesis permanente (Marcellesi 2003: 158). En consecuencia, los objetos de la mirada glotopolítica se identifican por medio de términos tales como interacción verbal o discurso, que remiten explícitamente al lenguaje como acción. Conceptos como el de lengua o dialecto -que, de entrada, evocan la existencia de un sistema lingüístico autónomo con respecto a su materialización discursiva- caen también en el ámbito de interés de la glotopolítica, pero como construcciones que responden a las condiciones históricas precisas de su nacimiento y desarrollo. Nos distanciamos por tanto de las teorías que objetivan lenguaje como sistema que existe con independencia de las prácticas en que se manifiesta.

La glotopolítica toma también distancia con respecto a las teorías del lenguaje que privilegian su función referencial y comunicativa para atender, en aras de la visibilización de su condición política, a sus funciones performativas. El "tú" que usa un estudiante al dirigirse al rector de la universidad no solo significa [segunda personal singular] sino que señala a un actor concreto en un acto de habla concreto e interviene además en la negociación de identidades sociales jerárquicamente ordenadas. Al decir 
"todos los empleados y empleadas" la gerente de una empresa no solo se refiere a la presencia de personas del sexo masculino y femenino bajo su supervisión sino que pudiera estar tomando una posición en relación con políticas de reivindicación de la igualdad entre mujeres y hombres en el ámbito laboral. Al publicar un "diccionario del español de México" -como veremos más adelante- no solo se está representando una variedad del español hablado en este país sino que se pudiera estar afirmando el derecho de este país a gestionar el idioma autónomamente y la capacidad para hacerlo. En cada caso, el sintagma señalado ("tú", "los empleados y empleadas" y "diccionario del español de México") adquiere pleno sentido social en tanto que discurso, es decir, en tanto que aparece utilizado en contextos precisos y conectado a identidades y relaciones sociales siempre en proceso de negociación. A esta conexión nos referimos como indicialidad, la propiedad que hace que el valor de un enunciado no esté solo en su dimensión referencial sino en su capacidad para señalar elementos del contexto de la enunciación, indicar identidades sociales y construir relaciones entre los interlocutores (Hanks 2000).

La indicialidad está directamente conectada con otra propiedad del lenguaje que resulta también central para la glotopolítica: la normatividad ${ }^{5}$. Nótese que, al anclar el estudio del funcionamiento del lenguaje en la acción conjunta de la referencialidad y la indicialidad, se problematiza la transparencia semántica que se le supone a las lenguas, sobre todo a aquellas que están altamente codificadas. El pleno significado de los enunciados, como acabamos de decir, depende no de una relación biunívoca y fija entre significantes y significados sino de las condiciones - de orden pragmático, cultural, social, político- en que se producen. De ahí que los individuos hablen (o escriban) según su orientación hacia el contexto de la interacción, según el modo en que lo conciban y según cómo quieran posicionarse en relación con él. Al usar el lenguaje siempre orientamos nuestra producción verbal en función de un contexto de normas socialmente constituidas y esta orientación liga inexorablemente lenguaje y metalenguaje, es decir, la práctica lingüística a las expectativas que puedan existir sobre el modo en que van a ser interpretadas. Desde esta perspectiva, el lenguaje "no existe aparte del modo en que se concibe y se habla de él" (Joseph 2006: 20), $\mathrm{y}$ uno de los espacios donde por definición se habla del lenguaje y de su normatividad son las academias de la lengua. 


\section{LA POLÍTICA LINGÜÍSTICA PANHISPÁNICA}

En el presente artículo dirigiremos nuestra mirada glotopolítica hacia la Real Academia Española (RAE) y la Asociación de Academias de la Lengua Española (ASALE) ${ }^{6}$. Nos centraremos, en concreto, en las últimas dos décadas, es decir, en el periodo caracterizado por lo que las propias instituciones bautizaron como política lingüística panhispánica y describieron así:

Las funciones atribuidas tradicionalmente a las Academias de la Lengua consistían en la elaboración, difusión y actualización de los tres grandes códigos normativos en los que se concentra la esencia y funcionamiento de cualquier lengua y que aseguran su unidad: la Ortografía, el Diccionario y la Gramática. Hasta hace algunos años, el modo de alcanzar esos objetivos se planteaba desde el deseo de mantener una lengua 'pura', basada en los hábitos lingüísticos de una parte reducida de sus hablantes, una lengua no contaminada por los extranjerismos ni alterada por el resultado de la propia evolución interna. En nuestros días, las Academias, en una orientación más adecuada y también más realista, se han fijado como tarea común la de garantizar el mantenimiento de la unidad básica del idioma, que es, en definitiva, lo que permite hablar de la comunidad hispanohablante, haciendo compatible la unidad del idioma con el reconocimiento de sus variedades internas y de su evolución (ASALE 2004: 3).

Vemos que esta narrativa de autorrepresentación ancla la acción estandarizadora en un sujeto plural ("las academias") que se manifiesta en torno a un punto de inflexión ("hasta hace algunos años" versus "en nuestros días"): hay un antes en la vida de estas instituciones, caracterizado por la defensa de la pureza del idioma y el protagonismo de la RAE, y un después (se puede considerar que la Ortografía de 1999 da el pistoletazo de salida) en que el propósito es salvaguardar la unidad por medio del trabajo

6 La RAE se creó en 1713 y, por iniciativa de esta, a partir de 1870, se empezaron a crear academias correspondientes de la Española en América. En 1951 se celebró en México el primer congreso que las reunió a todas y, aunque la Española no asistió por prohibición gubernamental, los congresos continuaron, ya con participación de la RAE, y fue cristalizando la Asociación de Academias de la Lengua Española. Véase García de la Concha (2014) y Zamora Vicente (1999) para la historia institucional. En Del Valle (2013) se trata el primer congreso y los efectos de la ausencia española. 
colectivo y el abrazo de la diversidad. En otras palabras, aunque la defensa de la unidad y prestigio del idioma ha estado siempre entre los objetivos de la RAE, lo que singulariza la fase panhispánica que se inaugura a finales del siglo veinte es la acción mancomunada y la normatividad policéntrica: toda iniciativa normativa se describe como intervención no de la RAE sino de la RAE y la ASALE y la protección de la unidad lingüística se afronta no desde el purismo y la imposición de la uniformidad sino, al contrario, desde el reconocimiento del cambio y la alabanza de la diversidad.

Subrayemos, en la imagen académica que emerge del fragmento citado arriba, que la unidad del español es condición imprescindible para la existencia de una comunidad panhispánica. Y es aquí donde, como hemos argumentado en trabajos previos (Del Valle y Gabriel-Stheeman 2004; Del Valle 2007), se halla la clave para la interpretación ideológica de las representaciones de la lengua -y de las instituciones que por ella velanque sostienen estas políticas: la declarada voluntad de ponerse al servicio de un proyecto de construcción de comunidad liga la acción académica de los últimos años al histórico movimiento panhispanista (Del Valle 2011; Sepúlveda 1994). Es precisamente esta versión del panhispanismo como estrategia de posicionamiento geopolítico de España y la centralidad que el movimiento le confirió a la lengua lo que nos invita a analizar la producción ya desde el último tercio del diecinueve de un sistema lingüístico-ideológico al cual nos referimos como hispanofonía: un conjunto articulado de representaciones del español que cobra sentido al ser leído ideológicamente en relación con un proyecto político asociado a la construcción de un imaginario nacional español y panhispánico ${ }^{7}$.

El contexto en que el panhispanismo y la hispanofonía se movilizan a finales del siglo veinte y principios del veintiuno lo hemos trabajado en los textos ya citados y no nos extenderemos aquí en presentarlo. Señalaremos solo que está asociado a la integración de España en la órbita atlántica (OTAN) y europea (UE) de los años ochenta, a la formación de grupos empresariales fuertes en el mismo periodo y a la proyección del capital español hacia los liberalizados mercados latinoamericanos a partir de los noventa. Es este contexto el que le confiere sentido al esfuerzo de la RAE por fortalecer el vínculo con las academias americanas, la colaboración de todas en la actividad normativa y, muy especialmente, su compromiso con la producción de nuevas representaciones de la lengua que participen más

7 El concepto de ideología hispanofónica (Del Valle 2007) deriva de trabajos sobre ideologías lingüísticas tales como Schieffelin, Woolard y Kroskrity (1998) y Joseph y Taylor (1990). 
felizmente que en el pasado en la colocación estratégica de España ante sus intereses en la América hispanohablante.

\section{LA LEGITIMIDAD NORMATIVA DE LAS ACADEMIAS: DESCRIPTIVISMO, AUTORIDAD Y CONSENSO}

Aunque son muchas las zonas del archivo por las que ingresar al análisis de las representaciones de la lengua española producidas en la formulación e implementación de la política lingüística panhispánica, la más obvia es quizás el conjunto de textos codificadores producidos por las academias desde que adoptaron esta política. Para el presente artículo hemos revisado los siguientes: la Ortografía (RAE, 1999), el Diccionario panhispánico de dudas (RAE y ASALE 2005), la Nueva gramática de la lengua española (RAE y ASALE 2010a), la nueva Ortografía de 2010 (RAE y ASALE 2010b), un manual académico para el buen uso del idioma (RAE y ASALE 2013) y la historia de la Academia escrita por su exdirector Víctor García de la Concha (2014). Si bien los textos en sí y la norma que proponen son centrales para comprender la propuesta panhispánica, también lo es la estructura paratextual que arropa a cada uno de ellos, en tanto que nos ofrece una serie de expresiones metalingüísticas en las que se puede apreciar el modo en que las academias quieren ya no solo representar la lengua cuya custodia asumen sino también desplegar una estrategia de autorrepresentación que las autorice y les confiera legitimidad.

Como ya señalamos, el aspecto acaso más evidente y previsible del discurso que emerge de estos textos es la orientación del conjunto de academias y su esfuerzo normativo hacia la idea de "unidad en la diversidad", lema oficioso de la corporación y eje conceptual de las políticas lingüísticas panhispánicas. Sin embargo, de la lectura de los textos normativos recientes extraemos otras tres ideas que nos permiten profundizar en su análisis glotopolítico. En primer lugar, la actividad de las academias se presenta como respuesta a una demanda de los hablantes y como satisfacción de una necesidad expresada desde abajo.

En consecuencia, la labor académica se proyecta con vocación popular y ambición de llegar a todos los espacios, geográficos y sociales, de la comunidad hispanohablante. En segundo lugar, se expone reiterada y meticulosamente la estructura del sistema diseñado para facilitar la colaboración interacadémica. Con ello, además de fortalecer el perfil panhispánico, se proyecta la imagen de una compleja pero ordenada organización de profesionales de la lengua 
ocupados en la gestión de su normatividad. Tercero, se produce una norma que, por un lado, pretende representar la totalidad del idioma y que, por otro, emerge de un delicado equilibrio entre la responsabilidad normativa de la academia y el descriptivismo que asocia el proyecto a los protocolos de la ciencia del lenguaje.

Ya la Ortografía de 1999 apuntaba en estas direcciones. Para empezar, aunque tímidamente, hacía su aparición el panhispanismo: estaba aún firmada solo por la RAE, pero advertía en la portada de que se trataba de una "Edición revisada por las Academias de la Lengua Española". Además, el prólogo anunciaba las líneas que se habrían de seguir en los años siguientes:

Han sido muchos los hispanohablantes que en los últimos tiempos se han dirigido a la Real Academia Española solicitando aclaraciones de normas ortográficas, planteando dudas y sugiriendo, en fin, la conveniencia de presentar la Ortografía de un modo más sistemático, claro y accesible ... Los detallados informes de las distintas Academias han permitido lograr una Ortografía verdaderamente panhispánica (RAE 1999: XIII).

Es de justicia que las últimas palabras sean de gratitud a los lingüistas que han colaborado con nosotros y, sobre todo, a las Academias hermanas que, con su diligente, minucioso y esmerado trabajo de revisión, han enriquecido el texto y han sancionado esta obra como la Ortografía de la comunidad panhispánica (RAE 1999: XIX).

Más allá, por tanto, de la afirmación de la hermandad académica y su servicio a la comunidad panhispánica, se va perfilando desde este texto una relación orgánica que une a las academias entre sí y a estas con los hablantes. Es en este sentido que se justifica la publicación del manual ortográfico como respuesta accesible a la expresión de dudas y petición de aclaraciones por parte de los hablantes. Al mismo tiempo, vemos surgir un complejo agente normativo de lingüistas y academias que ofrecen "detallados informes" y realizan un "diligente, minucioso y esmerado trabajo de revisión".

Una expresión más llamativa aún de este modelo de acción normativa sería el Diccionario panhispánico de dudas (DPD) publicado en 2005 y firmado, ahora sí, por la RAE y la ASALE. La sección de presentación de este libro reproducía casi literalmente el inicio del prólogo a la Ortografía pero matizaba aún más la relación entre academias y hablantes:

Centenares de hispanohablantes de todo el mundo se dirigen a diario a la Real Academia Española, o a cualquier otra de las que con ella integran la Asociación de Academias de la Lengua Española, exponiendo sus dudas sobre cuestiones ortográficas, léxicas o gramaticales y pidiendo aclaración sobre ellas. Buena parte de esas personas conocen y 
consultan de ordinario alguno de los manuales de corrección idiomática, diccionarios de dudas o libros de estilo existentes, pero quieren oír de manera directa la voz propia de las Academias, que tienen secularmente reconocida la competencia de fijar la norma lingüística para el mundo hispánico (RAE y ASALE 2005: XI).

En el DPD, por tanto, no solo se legitima la acción normativa de las academias presentándola como satisfacción de una necesidad expresada por los hablantes sino que se ancla el poder de la institución en la voluntad de estos ("quieren oír") y en la autoridad que le han traspasado a la corporación a lo largo de los siglos ("secularmente reconocida"). Resulta de particular interés el modo en que el DPD define esta relación orgánica entre hispanohablantes y academias a través de la discusión de la norma:

La norma, que el Diccionario académico define como "conjunto de criterios lingüísticos que regulan el uso considerado recto", no es algo decidido y arbitrariamente impuesto desde arriba: lo que las Academias hacen es registrar el consenso de la comunidad de los hispanohablantes y declarar norma, en el sentido de regla, lo que estos han convertido en hábito de corrección, siguiendo los modelos de la escritura o del habla considerados cultos (RAE y ASALE 2005: XI).

Aquí se ha dado un paso más en la consolidación de las academias como custodios autorizados de la norma que representa el orden lingüístico en tanto que tal norma viene desde abajo - de los hispanohablantes que han quedado constituidos en "pueblo" del que emana el poder-y resulta de un proceso caracterizado por el consenso.

Pocos años después, cuando se publicó la Nueva gramática de la lengua española en 2010, se profundizó aun más en el fortalecimiento de esta estrategia y de la visión de la norma que conllevaba. Como siempre, se afirmó, por encima de todo, el panhispanismo: "No es solo una obra colectiva, resultado de la colaboración de muchos, sino también una obra colegiada, el último exponente de la política lingüística panhispánica" (RAE y ASALE 2010a: XL). Se declaró también de nuevo el compromiso con los hablantes y la voluntad de que todos se sintieran interpelados y a todos fuera accesible el texto gramatical: "Se ha entendido, en suma, que la mejor forma de que la gramática académica llegue a todos - El español de todo el mundo es su lema de presentación- es construir distintas versiones del texto y adaptarlas a los más variados destinatarios" (RAE y ASALE 2010a: XLVI). Se insistió también en la amplia, inclusiva y altamente profesionalizada organización que gestó, produjo, corrigió y aprobó la gramática: después del congreso de academias de 1998, se acabaron de 
constituir las comisiones especiales de gramática en cada academia e Ignacio Bosque (de la Española) fue designado ponente. Tras el congreso de 2002 se aprobaron las líneas fundamentales del nuevo texto gramatical y se creó una Comisión Interacadémica que coordinaría Guillermo Rojo (de la Española) y en la que estarían representadas las áreas lingüísticas establecidas por la ASALE (parafraseo de RAE y ASALE 2010a: XL).

Finalmente, se trata con detalle la norma a partir del reconocimiento de la ya mencionada tensión inherente a un texto que se propone como descriptivo y normativo a la vez: "ha existido siempre en el tratado académico cierta tensión entre teoría y norma, o entre descripción y prescripción" (XLII). En la sección titulada "Antecedentes y presentación", resulta evidente la impronta descriptiva de la Gramática:

Describir las construcciones gramaticales propias del español general, así como reflejar adecuadamente las variantes fónicas, morfológicas y sintácticas que una determinada comunidad puede considerar propias de la lengua culta, aun cuando no coincidan enteramente con las opciones favorecidas en otras áreas geográficas. Registrar aquellas variantes conversacionales de la lengua no estándar atestiguadas en el mundo hispánico, siempre que estén bien documentadas y tengan interés para la descripción de las estructuras morfológicas o sintácticas ... [ [ La gramática es] una obra detallada, incluso meticulosa, que atiende a las diferencias que se establecen en función de áreas dialectales, niveles de lengua y registros ... Presta especial atención a la descripción de las principales variantes fónicas, morfológicas y sintácticas de todas las áreas hispanohablantes, así como a los matices de significado y las condiciones de uso que caracterizan un gran número de construcciones (ASALE, énfasis en el original).

Parece difícil ser más inclusivo en la elaboración de la norma. Se representa la lengua a través de una compleja taxonomía de criterios organizativos y variedades lingüísticas: áreas dialectales, niveles del lenguaje, registros, condiciones de uso, el español general, el español culto y variedades conversacionales. El objetivo es documentarla en su totalidad y no dejar ni un resquicio de la lengua sin registrar en el mapa gramatical. Era tan intenso este deseo que los publicistas del nuevo libro encontraron la etiqueta ideal: "la gramática del español total"8.

8 En estos párrafos parafraseo la lectura de la Nueva gramática que propuse en Del Valle 2009. 
Sin embargo, como adelantábamos arriba, este compromiso descriptivo no está reñido con la función normativa de las academias. La Nueva gramática reitera lo afirmado en textos anteriores y se plantea como objetivo "dar respuesta a posibles dudas sobre cuestiones normativas". Pero este abrazo simultáneo de descriptivismo y normatividad impone exigencias notables al proceso de autolegitimación, ya que durante la elaboración de gramáticas de vocación normativa en las que se adopta el uso como criterio de selección de la variedad que servirá de base a la norma, resulta particularmente sensible y con frecuencia polémica la decisión sobre qué usos seleccionar. La RAE y la ASALE resuelven el dilema afirmando haber sido capaces de saltarse el proceso de selección. Nos dicen que se limitan a “"describir", "reflejar" y "registrar" la lengua en su totalidad. Sin embargo, aunque la base de la gramática sea la lengua en su totalidad y la acción del gramático una mera descripción, la normatividad no desaparece: el español no se representa simplemente como una estructura gramatical sino como un sistema de múltiples normas situacionales, locales y generales. Desde esta perspectiva, la norma pareciera emerger directamente del pueblo y ser, por lo tanto, previa a la participación académica en el proceso normativo.

Este modelo de norma y esta visión de la normativización se veían reforzados en el año 2013, cuando las academias dieron a luz un libro titulado El buen uso del español. A pesar del perfil más explícitamente normativo que exhibe el libro desde el título mismo, la publicación nos ofrecía un texto más que dejaba en evidencia no solo la vocación panhispánica y el reconocimiento del carácter policéntrico del español sino el deseo de las academias de exhibir su comunión con el pueblo. De nuevo se respondía a las dudas con voluntad abarcadora al declarar el libro "destinado a la inmensa mayoría, a todos los hablantes que experimentan dudas o incertidumbres ortográficas o gramaticales ante la lengua" (RAE y ASALE 2013: XVI). Pero, sobre todo, se afirmaba el origen popular y democrático de la norma que las academias benévolamente gestionan:

Aunque los juicios sobre la norma se asocian a imposiciones y preceptos, las academias adoptan siempre una actitud positiva. Son conscientes de que promover y educar es más efectivo que prescribir y censurar. Dan a conocer a los hablantes las normas que ellos mismos han aprobado en plebiscito cotidiano, porque tienen la seguridad de que "el buen uso del español" favorecerá su desarrollo personal y social (RAE y ASALE, 2013: XVI).

La cobertura que hizo el diario madrileño El País de la presentación resulta sumamente reveladora en este sentido. El titular anuncia "LA REAL ACADEMIA SE ECHA A LA CALLE" y la autora del artículo relata 
impecablemente -acaso con cierta ironía- el deseo académico de comunión con el pueblo:

Como nacientes estrellas del rock and roll, micrófono en mano y cruzando el estrado con esa energía propia de los subidones de adrenalina, los académicos de la Real Academia Española coquetean sin complejos con un lenguaje nuevo y quizá algo impropio: el del espectáculo de masas. ... ayer 300 jóvenes llegados en autobuses desde León y Madrid asistieron a un acto que pretendía no solo anunciar el nuevo bebé de la casa sino una nueva fórmula de comunicación entre los científicos de la lengua y los usuarios de la calle (Fernández-Santos 2013).

\section{LA POLÍTICA LINGÜÍSTICA PANHISPÁNICA Y LA ELISIÓN DE LO POLÍTICO}

Desde el marco glotopolítico que adoptamos, podríamos ahora realizar un análisis crítico que visibilizara las contradicciones internas y las debilidades factuales del régimen de normatividad creado por la RAE y la ASALE . Podríamos, pongamos por caso, reparar en que, por mucho que se insista en la relación de igualdad entre academias y en la autoría colectiva de todas las obras normativas, no es la ASALE quien las firma, sino la RAE y la ASALE, indicio claro de la persistencia de una estructura jerárquica en la que la Española ocupa lugar superior. Podríamos también interrogar esa supuesta igualdad a través del examen de los procedimientos de trabajo colectivo y a partir, por ejemplo, del hecho de que en la elaboración de la Nueva gramática fueron dos académicos de la RAE (Ignacio Bosque y Guillermo Rojo) quienes protagonizaron el esfuerzo creador y organizativo. Podríamos también cuestionar el inocente consenso que se atribuye a los hablantes como mecanismo de producción de un sistema de normas lingüísticas y (inspirados por Bourdieu 1982) señalar cómo ese sistema es uno entre muchos mecanismos que contribuyen a la reproducción de la desigualdad social y

\footnotetext{
9 Al hablar de un régimen de normatividad debemos reconocer la deuda debida a Paul V. Kroskrity y al título del libro sobre ideologías lingüísticas por él editado: Regimes of Language. Optamos por la utilización de este término por sugerir la inscripción de la norma y de su elaboración en un espacio político, es decir, en un orden de poder y en un juego de distribución desigual de recursos (en este caso, lingüísticos).
} 
económica. Podríamos, finalmente, someter a examen la representatividad, autoridad y legitimidad de las academias revisando con cuidado los procesos por medio de los cuales fueron creadas y se reproducen ${ }^{10}$. ¿Quién, cuándo y por qué se crean las academias? ¿Quién y cómo elige a sus miembros? ¿Quién y cómo las autoriza? ¿Son estos protocolos propios de una organización que se autorrepresenta como democrática?

Es precisamente esta última interrogante la que más nos interesa en esta ocasión porque, en los discursos por medio de los que se describe y legitima el régimen lingüístico académico hallamos una inquietante reproducción (inquietante en vista de las contradicciones apuntadas en el párrafo anterior) de las estructuras conceptuales generales de la democracia liberal. Recordemos que el orden lingüístico representado por la norma académica es producido -se nos dice- por los propios hispanohablantes, quienes, a la vez, autorizan a las academias para gestionar y proteger ese orden. Notemos también que la producción de la norma ocurre -según las narrativas expuestas- por consenso democrático, el mismo consenso que informa la gestión y protección de la norma que llevan a cabo las academias. Estamos ante una réplica del concepto liberal de la política que va emergiendo en el tránsito de la antigüedad a la modernidad: el orden político no tiene ya su origen en la superioridad racional del rey-filósofo (o en la moral del monarca absoluto) sino que se proyecta desde abajo en un proceso de autorización - de alienación de los derechos individuales en una institución de gobierno- que requiere de un espacio público para el ejercicio de la negociación y el consenso (Mackenzie 2009: 21-50).

Notemos además, como decíamos arriba, que las academias parecen poco preocupadas por examinar el modo en que se genera la norma (apenas la describen, reflejan y registran) y lo interpretan sin más como una suerte de libre mercado lingüístico en el cual los intercambios proceden sin traumas apenas regulados por las prácticas consensuales de los hablantes y la benévola gestión de las academias. En este régimen lingüístico, la dimensión política de la lengua reside, por un lado, en la evidencia de las relaciones que existen entre las academias y otras organizaciones que se inscriben en el ámbito de la acción gubernamental y, por otro, en la lógica de la autorización desde abajo y en la práctica del consenso.

En un libro aparecido en los primeros meses de 2014, Víctor García de la Concha -director de la RAE entre 1998 y 2010 y del Instituto Cervantes a partir de 2012- repasa la historia de la institución y dedica un capítulo a 
la política lingüística panhispánica (en cuyo diseño e implementación fue él mismo una figura trascendental). Dos aspectos de ese capítulo merecen ser subrayados en el contexto del desarrollo del presente argumento. En primer lugar, describe en detalle el anclaje de la acción académica en el terreno de la política gubernamental:

Todas ellas [las 46 visitas institucionales realizadas a América entre 1999 y 2010] contaban con el respaldo personal de S. M. el Rey y el apoyo de nuestras embajadas e incluían un programa cuidadosamente preparado, con sesiones de trabajo en las Academias, visitas a los jefes de Estado, reuniones con los titulares de diversos ministerios, así como encuentros con los máximos responsables de las universidades de cada país -apoyo imprescindible para la labor académica- y de los medios de comunicación a fin de dar visibilidad social a esa tarea (García de la Concha 2014: 361).

En segundo lugar, da por zanjado cualquier pasado de disenso y explica la domesticación de la discrepancia por medio de la creación de un dispositivo académico abierto a todos y comprometido con el consenso (la palabra en sí aparece cinco veces en el capítulo):

Quedaban superadas viejas rencillas del pasado y, sin renunciar al debate y a la reivindicación de lo propio, se apostaba por una acción en común (García de la Concha 2014: 362).

Diccionarios, Gramática y Ortografía han sido y son el centro de atención de un trabajo mancomunado en el que todas las fases de elaboración de una obra nacen del consenso en el que se asienta la autoría común (García de la Concha 2014: 371).

La llamativa reiteración del consenso así como la ambición explícitamente totalizadora de las acciones académicas revelan lo que, en la lectura que aquí proponemos, es el fundamento ideológico más profundo de la política lingüística panhispánica: su efecto paradójicamente antipolítico, su impronta erradicadora de la tensión constitutiva de la norma lingüística y de la comunidad que en ella se pretende anclar. De cara a la realización de este análisis, nos han inspirado ciertos aspectos de la obra de la politóloga Chantal Mouffe, concretamente su crítica de lo que denomina el Zeitgeist pospolítico, la distinción que propone entre la política y lo político y la centralidad que confiere a las relaciones conflictuales en el desarrollo de la democracia. En primer lugar, Mouffe se posiciona frente a las versiones contemporáneas del liberalismo que proclaman el fin -fundamentalmente tras la caída del muro de Berlín- de las confrontaciones ideológicas significativas y la reducción 
de la política a la mera gestión de un orden que garantice los derechos individuales y el funcionamiento del libre mercado:

Los conflictos partisanos pertenecen al pasado, y el consenso puede ahora obtenerse a través del diálogo. Gracias a la globalización y a la universalización de la democracia liberal, podemos anticipar un futuro cosmopolita que traiga paz, prosperidad y la implementación de los derechos humanos en todo el mundo (Mouffe 2007: 9).

En este modelo no existe un afuera del orden democrático liberal y de los intereses que representa. Toda discrepancia es interna y se resuelve por medio de un proceso consensual, ya esté caracterizado por la voluntad de maximización de beneficios de cada una de las partes que negocian o, en versiones menos mercantiles (en general asociadas con Jürgen Habermas), inspirado por una racionalidad comunicativa de base ética (Mouffe 2007: 20).

La distinción entre la política y lo político le permite a Mouffe visibilizar lo elidido por la mirada pospolítica. El conjunto de prácticas y discursos institucionales que ordenan la comunidad y velan por su funcionamiento (lo que se vendría a asociar comúnmente con el aparato del Estado y con espacios gubernamentales) son lo que Mouffe identifica como la política. El conjunto de situaciones en las que se manifiestan y visibilizan las exclusiones constitutivas del orden comunitario y las relaciones de antagonismo que generan se identifican, en cambio, como lo político.

La distinción nosotros/ellos, que es condición de la posibilidad de formación de las identidades políticas, puede convertirse siempre en el locus de un antagonismo. Puesto que todas las formas de la identidad política implican una distinción nosotros/ellos, la posibilidad de emergencia de un antagonismo nunca puede ser eliminada (Mouffe 2007: 23).

Las identidades políticas (como los fonemas) se definen por oposición. Al tiempo que surge una identidad, surge un espacio exterior a esa identidad y se generan jerarquías y condiciones para el funcionamiento de la colectividad que privilegian a unos excluyendo a otros (Mouffe 2000: 51-73; 2007: 15-40). En las versiones liberales de la política democrática, se elide con vocación hegemónica esa exclusión constitutiva y se lleva a cabo una constante negación de la existencia de sujetos colectivos alternativos que, al hacer visibles las limitaciones del consenso, puedan representar un desafío al orden comunitario establecido.

La típica comprensión liberal del pluralismo afirma que vivimos en un mundo en el cual existen, de hecho, diversos valores y perspectivas 
que -debido a limitaciones empíricas- nunca podemos adoptar en su totalidad, pero que en su vinculación constituyen un conjunto armonioso y no conflictivo. Es por eso que este tipo de liberalismo se ve obligado a negar lo político en su dimensión antagónica (Mouffe 2007: 17).

El reconocimiento del carácter fundamental de esta relación antagónica-que nunca se puede resolver y solo se puede, en el mejor de los casos, canalizar en una perenne articulación conflictual- es la base, en la obra de Mouffe, de un modelo de democracia alternativo al liberal.

Volvamos ahora al régimen de normatividad de la RAE y la ASALE. Hemos visto a lo largo del presente artículo la formación de un dispositivo institucional de gestión de la lengua y la meticulosa construcción de una norma lingüística que estas instituciones codifican en una serie de textos concretos. Hemos visto que las propias instituciones normativas se autorrepresentan y representan la norma que producen como emanados de la comunidad de hispanohablantes que autoriza a las academias y genera, por medio del consenso (de un plebiscito cotidiano), la norma que aquellas apenas registran y gestionan. Hemos visto la pretensión de totalidad, de representar a todo aquel que pertenezca o quiera pertenecer a la comunidad hispanohablante, y hemos notado que es precisamente la vía del consenso la que se pretende que legitime ese afán totalizador.

Frente a esta visión de la política de la lengua, proponemos aquí que la elaboración de todo sistema normativo - da igual que esté fundado en prácticas lingüísticas que se ordenan en una, dos o más lenguas- tiene su origen en operaciones de inclusión y exclusión, y se despliega imbricado en procesos de formación de imaginarios colectivos, por lo mismo, siempre disputados. Sugerimos también que se ha de cuestionar críticamente la representatividad democrática de las academias, que, por su naturaleza, son instituciones exclusivas (en la doble y a la vez unitaria acepción del término).

El hecho es que, frente a esta pretensión de representatividad total y armonía panhispánica, existen espacios glotopolíticos antagónicos donde se visibiliza el déficit democrático del régimen lingüístico académico. Nos referimos a espacios donde se hallan momentos políticos, episodios polémicos de ruptura de la ideología hisponofónica que se proyecta, ambiciosa, como pensamiento único de la lengua. Me referiré, para concluir, a dos de estos eventos.

En el año 2010, la Academia Mexicana de la Lengua (AML), correspondiente de la Española y miembro de la ASALE, publicó el Diccionario de Mexicanismos que había sido dirigido por Concepción Company. Este acontecimiento editorial ponía broche de oro a un proyecto que hundía sus raíces tanto en la historia lingüística de México como en la de 
la asociación de academias de la lengua. Tal empresa había sido concebida ya por los miembros originales de la institución mexicana tras su fundación en 1875 (Cifuentes 2013). Se trata de un proyecto que, aunque nace de uno de los órganos del complejo académico que proclama la igualdad panhispánica, resulta revelador de los puntos ciegos de su discurso de autorrepresentación: reproduce la estructura jerárquica del conjunto de academias, es decir, un sistema que sitúa a la Española en el centro de la organización y a cargo de coordinar la construcción de la norma de todos, y a las academias americanas en la periferia del sistema (pero sin salirse del mismo) a cargo del registro de particularidades excluidas de la norma central. El hecho es que este antiguo proyecto, que tan visible hacía la desigualdad inherente al modelo, se presentaba con orgullo en 2010, en plena era panhispánica de discursos de igualdad y consenso que ya hemos descrito. Pero al punto de ruptura no se llegaría solo por lo visible de esta contradicción sino por una operación de elisión ideológica extrema perpetrada en el prólogo al afirmar su autora: "Es, hasta donde la Academia tiene noticia, el primer intento por recoger el léxico cotidiano del español actual, hablado y escrito de México" (Academia Mexicana de la Lengua 2010: XVI). Se excluía de esta manera tan simple el monumental proyecto coordinado por Luis Fernando Lara, del Colegio de México, y su resultado, el Diccionario del español de México (http://dem. colmex.mx/). Se trataba, insistimos, de una elisión ideológica extrema, a la cual así nos referimos porque, en su exceso, acababa revelando precisamente aquello que pretende ocultar ${ }^{11}$. Lara decidió manifestarse, salir a la carga y, en la revista cultural Letras libres, denunciar el ninguneo del que habían sido objeto su institución -el Colegio de México-, él y su equipo:

Sorprendente afirmación, si consideramos que los filólogos mexicanos que forman parte de la Academia conocen suficientemente el estudio que, sobre la base de los dos millones de apariciones de palabras del Corpus del español mexicano contemporáneo (1921-1974), ha venido dando lugar a una serie de diccionarios que en noviembre de 2012 se coronaron con el Diccionario del español de México (El Colegio de México), el segundo diccionario integral del español basado en una concepción nacional -el primero fue el Diccionario integral del español de Argentina, Buenos Aires, Tinta Fresca, 2008- y no periférica de la lengua. Un vocablo que aparentemente debe el español a los mexicanos es ningunear: Concepción Company y sus colegas de la Academia se muestran muy hábiles en

11 El concepto de elisión ideológica corresponde al de "erasure" que, en inglés, proponen Irvine y Gal (2000). 
el ninguneo del conjunto de estudios y publicaciones del equipo del Diccionario del español de México (Lara 2011a: 69).

Aprovechó, además, Lara la oportunidad para cuestionar el concepto de mexicanismo que la AML definía por contraste con el "español peninsular, concretamente, de su variedad castellana" (Academia Mexicana de la Lengua 2010: XVI). ¿Qué sentido tiene examinar el léxico mexicano por contraste solo con la variedad castellana? Si de hacer una comparación de todas las variedades del español se tratara, tendría más sentido lexicográfico, decía Lara, compararlas todas en pie de igualdad. El arreglo actual que se revelaba en el Diccionario de mexicanismos suponía la reproducción de una estructura organizativa de perfil neocolonial:

Por lo que se ve, ni a la Academia Mexicana, ni a la Española y las demás les interesa una comparación de esta clase. Más bien se trata de perpetuar, en la conciencia de los hispanohablantes, la distinción entre un español metropolitano y los españoles coloniales... impulsando la publicación de diccionarios de americanismos, peruanismos, etcétera, sin contar con suficientes datos comparativos (Lara 2011a: 69).

Denunciaba así el servilismo de la AML y la lógica centro-periferia que sostenía el régimen de normatividad que las academias correspondientes se prestan a legitimar. La respuesta de Lara dio inicio a una fascinante polémica, principalmente librada entre él mismo y Company, que duró varios meses ${ }^{12}$. Pero valga esta breve exposición para hacer notar este momento antagónico que, materializado en espacio tan prominente de la esfera pública como Letras Libres, visibilizó la autonomía de las instituciones mexicanas (en este caso el equipo del Colegio de México) con respecto al sistema de academias y su plena capacidad para gestionar la lengua desde el cierre nacional y al margen, completamente al margen, de las adherencias institucionales que conlleva el ideologema de la unidad en la diversidad.

El segundo evento al que nos vamos a referir se produce en España en marzo de 2012. Ignacio Bosque, miembro destacado de la RAE -ya mencionado arriba por su papel rector en la elaboración de la Nueva gramática-, hacía público a través del ya citado diario El País un texto titulado "Sexismo lingüístico y visibilidad de la mujer" y presentado como perteneciente al género del informe. El texto, en el momento de su

12 El primer artículo crítico de Lara aparece en la bibliografía como Lara (2011a), la respuesta de Company como Company (2011a), la nueva réplica de Lara como Lara (2011b). Otra crítica es Zaid (2011) y una nueva réplica de Company es Company (2011b). 
publicación, había sido suscrito por todos los académicos de la RAE, de número y correspondientes, presentes en la sesión del 1 de marzo de ese año y consistía en una agresiva impugnación de una serie de guías y manuales que, en los últimos años de la primera década del siglo veintiuno, se habían publicado promoviendo usos no sexistas del lenguaje. Citaremos, a modo de orientación, algunos títulos: Guía para un uso del lenguaje no sexista en las relaciones laborales y en el ámbito sindical. Guía para delegadas y delegados (publicado por la Secretaría Confederal de la Mujer de Comisiones Obreras y por el Ministerio de Igualdad en 2010); Guía de uso no sexista del lenguaje de la Universidad de Murcia (publicado por la Unidad para la Igualdad entre Mujeres y Hombres de la Universidad de Murcia en 2011); Guía sindical del lenguaje no sexista (publicado por la Secretaría de Igualdad de la Unión General de Trabajadores en 2008); Igualdad, lenguaje y Administración: propuestas para un uso sexista del lenguaje (publicado por la Conselleria de Bienestar Social de la Generalitat Valenciana en 2009).

No deja de ser notable el hecho de que, a pesar de la ubicuidad de prácticas de higiene verbal (Cameron 1995) mayormente ajenas a la Academia, fuera precisamente ésta la que la RAE decidiera convertir en objeto merecedor de un ataque tan abierto. Estas guías y manuales son manifestaciones concretas de la higiene verbal en las cuales la práctica normativa viene asociada a la reivindicación de igualdad por parte de un colectivo históricamente discriminado. Lo revelador del caso y de la polémica que siguió a la aparición del informe - a efectos de la presente argumentaciónes la aparente emergencia de un complejo agente glotopolítico conformado por movimientos feministas, sindicatos de izquierdas, universidades y espacios institucionales a nivel local y de comunidad autónoma. A través de los títulos y de las organizaciones que asumen la publicación de estas guías, se vislumbra la emergencia de un régimen de normatividad y una institucionalidad de la lengua completamente ajena al entramado académico y a su ideología hispanofónica. Y ahí radica acaso la ruptura ante la cual la Academia siente que no puede abstraerse y opta por atacar confiando, suponemos, en su capacidad de victoria sobre este adversario particular ante la opinión pública. Pero esa victoria -y no estamos en posición de juzgar si se produjo o no- resulta irrelevante, pues la ruptura está en la afirmación de la inconmensurabilidad de ambos proyectos, en la articulación de un antagonismo que no acepta las reglas del juego hegemónicas del discurso panhispánico y fuerza a las instituciones que lo producen a desvelar lo político de su condición, su necesario anclaje en operaciones de exclusión. 


\section{CONCLUSIÓN}

En este artículo, partimos situándonos en el marco teórico-metodológico de la glotopolítica y, al hacerlo, reivindicamos una concepción generosa de la tipología de situaciones en que se manifiesta la condición política del lenguaje. No solo en las acciones del aparato del Estado y de las organizaciones gubernamentales sobre la lengua se observa esta condición, sino también en un amplio espectro de discursos y procesos de interacción verbal por medio de los cuales se constituyen subjetividades e identidades colectivas relevantes para el orden político de una comunidad. Desde esta perspectiva, proyectamos nuestra mirada hacia la política lingüística panhispánica diseñada e implementada por la RAE y la ASALE.

Así, visitamos acciones institucionales sobre la lengua -al discutir la estructura de las academias y los protocolos que siguen en su gestión de la normatividad-y nos acercamos también a los discursos producidos en el proceso - examinando, principalmente, la estructura paratextual de las obras codificadoras-. Más allá de la lectura ofrecida en trabajos anteriores - en los que habíamos relacionado la política lingüística panhispánica con el desarrollo del nacionalismo español y asociado el panhispanismo con discursos y prácticas neocoloniales- exploramos en este artículo el anclaje ideológico de las academias - tanto del modo en que representan la norma como el modo en que se representan a sí mismas- en la filosofía política liberal.

Vimos que las academias se presentan explícitamente como legítimas gestoras del idioma autorizadas por los hispanohablantes y comprometidas con la acción mancomunada basada en el consenso. Vimos además que el desarrollo de la norma se les atribuye a los propios hispanohablantes, quienes proceden también al hacerlo de acuerdo con el consenso democrático. Las academias serían simples registradoras de una norma que emerge de la comunidad hispanohablante. Nuestro análisis reveló, en definitiva, que la política lingüística panhispánica se fundamenta ideológicamente en una concepción consensual de la política y en una declarada fe en el potencial normalizador y unificador de la acción combinada de la racionalidad gramatical y de la buena voluntad.

Finalmente, pusimos el pie en el espacio del antagonismo donde se revela la condición precaria e inestable de todo orden social y, en el caso que nos ocupa, de todo régimen de normatividad lingüística. En la última sección, visitamos brevemente dos polémicas que dejan en evidencia los límites de la política lingüística panhispánica al hacer visible el hecho de que esta opera inevitablemente por medio de exclusiones. Concluimos que es posible la 
existencia de un afuera de la política lingüística panhispánica y que surge inexorablemente en toda oportunidad en que la exclusión se hace extrema. Concluimos que es un hecho la construcción de imaginarios alternativos de la lengua anclados ya sea en proyectos que, como el de Luis Fernando Lara en El Colegio de México, reivindican la soberanía nacional, o que responden, como en el caso de las guías para el uso no sexista del lenguaje, a las inquietudes de movimientos de resistencia a la discriminación.

Acaso deba ser una de las misiones centrales de la glotopolítica el reconocimiento de lo político del lenguaje - de la condición antagónica de todo régimen de normatividad lingüística y, por ende, de toda comunidad-. Acaso ese reconocimiento deba ser el eje articulador de políticas lingüísticas orientadas a la construcción de un espacio público comunicativo polifónico que negocie la paradoja original consciente de la incapacidad de resolverla y que sospeche, siempre, de formulaciones unívocas de la democracia como consenso que conduce a la unidad total y perfecta.

\section{REFERENCIAS BIBLIOGRÁFICAS}

Academia Mexicana de la Lengua. 2010. Diccionario de mexicanismos. México: Siglo XXI. Arnoux, Elvira Narvaja DE. 2000. La glotopolítica: transformaciones de un campo disciplinario. En Alfredo Rubione (coord.). Lenguajes: teorías y prácticas, pp. 15-42. Buenos Aires: Gobierno de la Ciudad de Buenos Aires e Instituto Superior del Profesorado. ASALE. 2004. La nueva política lingüistica panhispánica. Madrid: Real Academia Española. BosQue, IgnaCio. 2012. Sexismo lingüístico y visibilidad de la mujer [en línea]. Disponible en http://www.rae.es/sites/default/files/Sexismo_linguistico_y_visibilidad_de_la_mujer_0. pdf [Consulta 28/7/2014].

Bourdieu, Pierre. 1982. Ce que parler veut dire; L'economie des echanges linguistiques. París: Fayard.

Cameron, Deborah. 1995. Verbal Hygiene. Londres/Nueva York: Routledge.

Cifuentes, BÁRbara. 2013. The politics of lexicography in the Mexican Academy in the late nineteenth century. En José del Valle (ed.). A Political History of Spanish: The Making of a Language, pp. 167-181. Cambridge: Cambridge University Press.

Company, Concepción. 2011a. Un diccionario a debate. Letras libres (abril de 2011): 50-53. 2011b. El Diccionario de mexicanismos frente a los críticos. NEXOS 1 de julio de 2011 [en línea]. Disponible en http://www.nexos.com.mx/?p=14353.

Del Valle, José. 2007. La lengua, ¿patria común? Ideas e ideologías del español. Fráncfort y Madrid: Vervuert e Iberoamericana.

2009. Total Spanish: the politics of a Pan-Hispanic grammar. PMLA 124(3): 880-886.

2011. Panhispanismo e hispanofonía: breve historia de dos ideologías siamesas. Sociolinguistic Studies 5 (3): 465-484. 
2013. Linguistic emancipation and the academies of the Spanish language in the twentieth century: the 1951 turning point. En José del Valle (ed.). A Political History of Spanish: The Making of a Language, pp. 229-245. Cambridge: Cambridge University Press

Del Valle, José y Luis Gabriel-Stheeman. 2004. La batalla del idioma: la intelectualidad hispánica ante la lengua. Fráncfort y Madrid: Vervuert e Iberoamericana.

Irvine, Judith T. y Susan Gal. 2000. Language ideology and linguistic differentiation. En Paul V. Kroskrity (ed.). Regimes of language, pp. 35-84. Santa Fe: School for American Research.

Fernández-Santos, Elsa. 2013. La Real Academia se echa a la calle. El País [en línea], 13 de diciembre de 2013.

García de la Concha, Víctor. 2014. La Real Academia Española: vida e historia. Barcelona: Espasa.

Guespin, Louis y Jean-Baptiste Marcellesi. 1986. Pour la glottopolitique. Langages 83: 5-34.

Hanks, William. 2000. Indexicality. En Alessandro Duranti (ed.). Language Matters in Anthropology: A Lexicon for the Millenium, número especial de Journal of Linguistic Anthropology 9(1/2): 124-126.

Haugen, Einar. 1972. The Ecology of Language. Stanford: Stanford University Press.

Joseph, John E. 2006. Language and politics. Edimburgo: Edinburgh University Press.

Joseph, John E. y TALBOT J. TAYLOR (eds.). 1990. Ideologies of language. Londres y Nueva York: Routledge.

KAPLAN, Robert B. y Richard B. BALDAUF JR. 1997. Language planning from theory to practice. Clevedon: Multilingual Matters.

Kroskrity, Paul V. (ed.). 2000. Regimes of language: ideologies, polities, and identities. Santa Fe: School of American Research Press.

Lara, Luis Fernando. 2011a. El "mexicanismo" de la Academia de la Lengua. Letras libres (febrero de 2011): 68-72.

2011b. De nuevo sobre los mexicanismos y su identidad. Letras libres (abril de 2011): 54-56.

Mackenzie, IAin. 2009. Politics. Londres y Nueva York: Continuum.

Marcellesi, Jean-Baptiste. 2003. Glottopolitique: ma part de verite. Glottopol 1: 156-158.

Martin-Jones, MariLYn. 1989. Language power and linguistic minorities: the need for an alternative approach to bilingualism, language maintenance and shift. En Ralph Grillo (ed.). Social anthropology and the Politics of Language, pp. 106-125. Londres y Nueva York: Routledge.

Maurais, Jacques y Michael A. Morris (eds.). 2003. Languages in a globalising world. Cambridge: Cambridge University Press.

Mouffe, Chantal. 2000. La paradoja democrática: el peligro del consenso en la política contemporánea. Barcelona: Gedisa.

2007. En torno a lo político. Buenos Aires: Fondo de Cultura Económica.

Real Academia Española. 1999. Ortografía de la lengua Española. Madrid: Espasa.

Real Academia Española y Asociación de Academias de la Lengua Española. 2005. Diccionario panhispánico de dudas. Madrid: Santillana.

2010a. Nueva gramática de la lengua española. Barcelona: Planeta.

2010b. Ortografía de la lengua española. Madrid: Espasa.

2013. El buen uso del español. Madrid: Espasa.

Schieffelin, Bambi B., Kathryn Woolard y Paul V. Kroskrity (eds.). 1998. Language ideologies: practice and theory. Nueva York: Oxford University Press,

Senz, Silvia y Montserrat Alberte (eds.). 2011. El dardo en la Academia: esencia y vigencia de las academias de la lengua española. 2 volúmenes. Barcelona: Melusina. 
Sepúlveda, Isidro. 1994. Comunidad cultural e hispano-americanismo, 1885-1936. Madrid: Universidad Nacional de Educación a Distancia.

2005. El sueño de la Madre Patria. Hispanoamericanismo y nacionalismo. Madrid: Marcial Pons.

TAYLOR, TALBOT. 1997. Theorizing language: analysis, normativity, rhetoric, history. Amsterdam, Nueva York y Oxford: Pergamon.

ZAID, GABRIEL. 2011. La mala suerte. Letras libres (abril de 2011): 32-34.

Zamora Vicente, Alonso. 1999. Historia de Real Academia Española. Madrid: Espasa. 